\title{
¿Cuán útill es para un oftalmólogo saber Histopatología?
}

\section{Is Histopathology really needed for the ophthalmologist?}

\author{
Sonia Corredor-Casas* y Van Charles Lansingh \\ Servicio de Patología, Instituto Mexicano de Oftalmología, Querétaro, Querétaro, México
}

Estimado editor:

Frecuentemente, la especialidad de Patología en Oftalmología se ve desdeñada en nuestro país. Prueba de ello es el hecho de que solamente existen 8 personas con entrenamiento formal $y$, desafortunadamente, un puñado más que trabajan en el extranjero, donde buscan nuevas oportunidades y desarrollo profesional, que es muy difícil poder proporcionar en México.

Sin embargo, como en pocas especialidades, el conocimiento adecuado de la anatomía microscópica es esencial para el oftalmólogo, a fin de lograr un mejor entendimiento de cómo y dónde realizar procedimientos quirúrgicos, así como de la fisiología que permite que ciertos medicamentos funcionen de un modo $u$ otro, y de esta manera elaborar la mejor propuesta terapéutica en cada caso. En la práctica, muchos colegas tienen que enviar las muestras que desean analizar a laboratorios donde no se cuenta con el entrenamiento adecuado para interpretar los hallazgos presentes y suministrar información de verdadera utilidad, lo cual también resulta en una formación menos completa para nuestros futuros colegas. Además, puede verse afectada la toma de decisiones en el manejo de los pacientes.

Es por esto que en el IMO (Instituto Mexicano de Oftalmología), desde que se instituyó el programa de residencia, hace casi 8 años, se procuró contar con este servicio complementario a la especialidad. Este logro se cristalizó hace 4.5 años y ha requerido un esfuerzo en ocasiones titánico. Afortunadamente, hoy podemos decir que, junto con la atención del paciente 300 mil, también celebramos el logro de haber realizado el pasado 27 de agosto el estudio histopatológico de la biopsia número 1000 en nuestro laboratorio de patología. Desde febrero de 2014 hasta la fecha, hemos tenido un crecimiento exponencial, y en estos 8 meses del 2018 hemos alcanzado las 200 biopsias estudiadas.

En este recorrido de casi 5 años hemos sido testigos de un cambio radical en la mentalidad de nuestros oftalmólogos graduados y en entrenamiento, quienes han asumido completamente la responsabilidad de enviar para su estudio microscópico todas y cada una de las muestras de tejido que obtienen en los procedimientos diarios realizados a los pacientes del instituto. Dentro de las muestras analizadas en el laboratorio, están desde extendidos celulares de lesiones de superficie ocular, hasta biopsias líquidas intraoculares, pasando por resecciones de masas orbitarias, y tal vez lo más importante, tejidos habitualmente desechados en la mayoría de nuestros hospitales, como son los productos de curetaje con diagnóstico clínico de Chalazión, los productos de evisceración y los «menospreciados» pterigiones. Con respecto a este último grupo de muestras, vale la pena mencionar que, gracias a que desde hace tres años se encontró en nuestro

Correspondencia:

*Sonia Corredor-Casas

Avenida Estadio Sn

Col. Centro Sur, Del. Colinas de Cimatario

C.P. 76090, Santiago de Querétaro, Fecha de recepción: 12-09-2018

Fecha de aceptación: 10-10-2018

Disponible en internet: 02-01-2019

Qro., México

DOI: 10.24875/RMO.M18000056

Rev Mex Oftalmol. 2019;93(1):46-47 www.rmo.com.mx

0187-4519/○ 2018 Sociedad Mexicana de Oftalmología. Publicado por Permanyer México. Este es un artículo Open Access bajo la licencia CC BY-NC-ND (http://creativecommons.org/licenses/by-nc-nd/4.0/). 
hospital, en un estudio piloto a punto de ser publicado, una frecuencia cercana al $12 \%$ de cambios precursores de malignidad Neoplasia de la Superficie Ocular, NESO en estos tejidos, se han redoblado los esfuerzos e instaurado un nuevo protocolo para tratar de detectar tempranamente la presencia de displasia tanto clínicamente como en los productos de la cirugía de pterigión, lo que ha llevado a intervenciones más oportunas y a mejorar el pronóstico, la morbilidad y la calidad de vida de estos pacientes. En la actualidad, nuestros residentes tienen un curso completo de Patología Oftálmica, que abarca cada uno de los segmentos del globo ocular y pretende que los estudiantes adquieran un conocimiento firme en este tema, evaluable en las pruebas nacionales e internacionales a las que se presentan.

Otras áreas de oportunidad en el campo de la Patología Oftálmica que estamos explorando actualmente en el instituto son aquellas relacionadas con el estudio e implementación de la respuesta tisular a variantes de tratamientos médicos (uso de fármacos, moléculas alternativas, etc.) o relacionadas con propuestas de modificaciones en técnicas quirúrgicas que supongan una mayor accesibilidad para el paciente con una mejor relación costo-beneficio (por ejemplo, dispositivos intraoculares 0 instrumentos quirúrgicos con diseño óptimo, pero más económicos que los existentes actualmente en el mercado), los cuales constituyen protocolos en curso en asociación con los departamentos de Segmento Anterior y Retina.

De forma adicional, en el campo del estudio de factores de riesgo asociados a hábitos presentes en nuestras regiones geográficas, como pueden ser los ocupacionales, alimentarios y/o tóxicos medioambientales, recientemente nos hemos dado a la tarea de estudiar tanto los efectos de la presunta mayor radiación ultravioleta presente en nuestra zona (Bajío), como a determinar de manera bastante aproximada la cantidad de proteína presente en muestras representativas de los tipos de comida rápida más comúnmente consumida por nuestra población. Esto último motivado por el hecho de que desde hace ya varios años ostentamos el nada honroso primer lugar a nivel mundial en obesidad infantil, con el subsecuente riesgo aumentado para diabetes mellitus y sus manifestaciones oculares desde muy temprana edad. Estos dos trabajos son objeto de publicaciones en curso.

Dentro del programa de educación médica continua que promueve nuestro hospital he tenido la oportunidad de asistir durante un mes al Servicio de Patología Oftálmica del Hospital de la Universidad de Mc Gill en Montreal, donde he adquirido herramientas muy valiosas para la implementación de técnicas de laboratorio aplicables en nuestro medio, así como la actualización en el conocimiento de los avances más recientes en la especialidad. Además, se ha logrado establecer una estrecha colaboración con dicho laboratorio con fines académicos, para interconsulta de casos de interés, publicaciones y participación en eventos internacionales, entre otros.

A nivel nacional es de nuestro especial interés trabajar para la difusión del conocimiento de la Patología Oftálmica en toda la república, llevando a cabo participaciones en conjunto con asociaciones de subespecialidades, como la de Órbita del estado de Jalisco, con quienes compartimos una actualización en la «Utilidad de la Inmunohistoquímica» en las lesiones de esta localización hace algunas semanas en la ciudad de Guadalajara.

Sea esta la oportunidad para convocar a todos los colegas para cobrar conciencia acerca de la importancia de extraer toda la información posible a partir del estudio microscópico y por técnicas de laboratorio de cualquier muestra tisular obtenida de nuestros pacientes, conscientes de que es una oportunidad invaluable para realizar diagnósticos tempranos que aumenten la probabilidad de curación o mejoren el pronóstico para varias entidades. Ante esta perspectiva y en atención a la creciente necesidad de proteger el ejercicio de la práctica oftalmológica diaria de toda sombra de duda y evitar problemas médico-legales, sería de obligatorio cumplimiento en todos nuestros hospitales el estudiar todos los tejidos recuperados tanto en el quirófano como en el consultorio.

\section{Conflicto de intereses}

Los autores declaran que no existen conflicto de intereses. 